\title{
User Data Driven Recommendation for Location
}

\author{
Harini. M, Bala Krishna, Sanjay Bhargav
}

\begin{abstract}
Location recommendation plays a crucial play in serving to users to seek out their interested spots. Considering recent analysis that was studied the way to advocate locations with the important, social, geographical knowledge, a number of them shown regarding the problems relating to attributes of new users. complicated methodology is to convey them into explicit- feedback- based mostly content-aware (cf)collaborative filtering, however they have to draw negative attributes for higher learning performance, as users' negative preference isn't thought-about in human quality. Before theories have slightly shown sampling-based strategies don't exercising well. So, we tend to projected a ascendable Implicit-feedback-

based mostly Content-aware cooperative Filtering (ICCF) framework to urge precise real knowledge and to good afar from negative attributes sampling. At last, we tend to perform ICCF with in users have profiles and details of attributes. Final performance show that ICCF outperforms many different competitory baselines,so that user attribute info isn't solely effective for up recommendations however additionally addressing initial knowledge and different eventualities.
\end{abstract}

\section{Keywords- location, most visited, matrix filtering.}

\section{I.INTRODUCTION}

The popularity of travel primarily based product firms arrival, photos sharing created usually throughout travel in existing system, new somebody fix up travel supported anonymous user reviews and ratings (just dial, tripadvisor, makemytrip). in projected system, implicit-feedback-based cooperative filtering (cf) is one in all the foremost no-hit recommendation approaches to deal with vast knowledge in planet. Collaborative filtering ways equally treat each user and attribute, and might totally differentiate the variation of user's interests across different domains by matching the attributes. the most advantage of $\mathrm{cf}$ is to advocate the most effective place of interests (poi) within the cluster. iccf not solely improves location recommendation, however conjointly addresses the initial issues of each new users and locations, to induce the mapping procedure, we tend to created a replacement novel variable substitution technique to differentiate the training of iccf into two weighted least sq. issues with reference to user/location latent factors, and two multiple dependent-variable regression issues with reference to feature latent issue matrices. learning concerning user and site latent factors in weighted least sq. issues, Here we tend to projected coordinate attribute for optimisation, to that scales across linearly with knowledge and have size, and

Revised Manuscript Received on September 22, 2019.

Harini.M, SRM Institute of Science And Technology, Kattankulathur, Chennai harini.officialmail@gmail.com.

Bala Krishna, Srm Institute of Science And Technology, Kattankulathur, Chennaisaibala_r@srmuniv.edu.in.

Sanjay Bhargav, SRM Institute of Science And Technology,

Kattankulathur, Chennai. edarasanjaybhargav_ed@ srmuniv.edu.in. quadratically with the dimension of latent area,without any adjustment to the rule, with this we are able to simply confirm whether or not to incorporate user/location bias or not simply by combining user/location latent matrix with either associate all-one vector (or) associate all-zero vector. The inheritance of user/location bias which might any facilitate to combat with sparseness problems, from the empirical studies. Identification of High and traditional POIs. to induce the necessary real knowledge data from mechanical phenomenon knowledge, some extent 2 stage agglomeration manner is projected that depicts hot places of attention-grabbing (POIs) and traditional POIs, severally. in several to traditional approaches that determine solely high POIs, our POIs extraction approach higher represents the mechanical phenomenon and every one depicts the data loss. we've got described that the ultimate results might have similar characteristics and be stannant because of the actual fact that each one of the factors area unit collective into one score for every travel route. Parallely, the system can retrieve the top-k routes with the best search place because the results. Users might not perceive the essence of those paths through the ultimate single record.it should be troublesome to pick a path from the ultimate output results. Ensuing, users have to be compelled upon predefining the score for every issue, perhaps this exhausting to settle on an appropriate load under utmost circumstances. Since location path testimonial should think about many determinants into thought to stress the only voyage portions of touring means, we tend to used the idea of Range based Representative Skyline to retrieve journey methods. Objective based Representative Skyline quest above some tour plans conjointly holds a little variety $\mathrm{k}$ concerning outline maps that adequately express the complete optimal( output in phases of the output portrayed. So,in the knowledge wherever the count vector like POIs depicts the attractiveness rate and therefore the visiting time knowledge. To calculate the mean dish number, we tend to get the ultimate score values. as an instance, with $\mathrm{k} 3$, the outline points in are often divided into 3 subsets, and . Our emblematic outline tour path resolution can report $\mathrm{f}$,so once a user opt for a choice, the output usually depends each thereon user's own information \&data, choices, inclinations and ways further as literal/implied cultural sways of the surface surroundings, like suggestions of compatriots. though this outside human interplay is troublesome to view and portray undeviatingly, influences area unit in- directly mirrored over those shifting exemplars of familiar teams. any additional traverse particular taxi operator instance, time of a well-liked performance within town, the chance a cab operator can go path in to the location with next client is eminent. Finally aforementioned surface surroundings (i.e. any of the special event) will give hints to assist with location prediction. luckily,such outside characters may also be mirrored by cluster patterns. So, inturn outer 
interplays may be portrayed traversing numerous inducing trims during a cluster. At last, any gathered obvious interplay data is useful to unusual path step foresight.

\section{MODULES TRAVELER REGISTRATION:}

Identity management (ID management) could be a wide understanding path that contend with characteristic users during a system by attributes (considering a rustic, a network, or maybe associate enterprise) and dominant their gain of access to resources at intervals that system by considering user rights and restrictions with the user established identity. throughout identity management of the human, we have a tendency to acquire credentials like profession, school, college, operating organization name, favorite holidaymaker spots to get human number for every travelers. Admin login is employed to verify the review and holidaymaker places.

\section{SUGGESTION OF HOLIDAYMAKER PLACE SUPPORTED MULTIPLE:}

Also our projected system, provides results for multiple keywords by analyzing the geographic location, time and attribute. we have a tendency to contemplate a situation wherever users stipulate bents with keywords. as an instance, once designing visit in state capital, one possess "Opera House". As before-mentioned, we have a tendency to elongate information regarding tour designing by traversing potential keywords assigned through users.

Example:

Buffet - ECR - Lunch

Resort - Swimming - Sunrise. Breakfast - Boat - Lunch

\section{COLLABORATIVE FILTERING:}

Collaborative filtering, additionally taken as convivial filtering,filters information via mistreatment the info advice of others. it's supported the thought that folks United Nations agency united within their analysis of bound things within the past square measure seemingly to agree once more in the future. an individual United Nations agency interested to observe a film as an example, might contemplate for recommendations from colleagues/friends. Then recommendations of some friends United Nations agency have similar interests square measure trusty quite recommendations from random users/others. filtering server. currently the user have heap of suggestion, user must opt for cooperative filtering technique to filter the knowledge by mistreatment the advice of the chums. CF solely utilizes the user-item rating matrix to create predictions and proposals, avoiding the requirement for aggregation intensive info concerning things and users. additionally, CF will be simply adopted in numerous recommender systems without requiring any domain information. Given the effectiveness and convenience, several CF strategies are projected, that constitute 2 categories: rating primarily based \} and ranking based.

The every and each module within the given discovered paper we have a tendency to use it to see the places for holiday maker and information offer by his profile and
The user and place list square measure keep in cooperative

additionally together with his looking places by the order American state highest in ranking.

We investigate the way to consolidate prejudices into content conscious matrix resolution with none fitting to optimisation formula, enacted by the way to augment intrinsic factors of users and places. we have a tendency to additionally by trial and error study the consequences of inclinations and discern their vital profit to advice from distributed datasets.

\section{ELABORATES}

We impose a idea for building a secure bond of the projected model with a graph regularised matrix resolution. We develop a Keyword aware Representative Travel Route (KRTR) framework to reclaim sparse endorsed maps wherever keyword suggests that the customised elements that users possess for the journey. The way dataset can be designed from the gathering of low sampling arrival records. Three existing constant schemes for creating uninterested choice and generally includes a very distinctive mixed constant methodology. The effectiveness from the projected distributed and rank one constant plans has been widely estimated, showing its very important profit for rising recommendation, in particular for locations at rich ends.

We state associate economical correlative reduction optimisation formula to be told parameters within the distributed and rank one coefficient schemes, that ranges linearly by information size have size, and among the dimension of the underlying house. additionally, to a technical analysis of your time quality, we have a tendency to by trial and error study convergence and potency problems within the projected optimisation formula.

As of similar to the before represented in modules the user first register with his attributes and then moves on to the next section of searching for places.

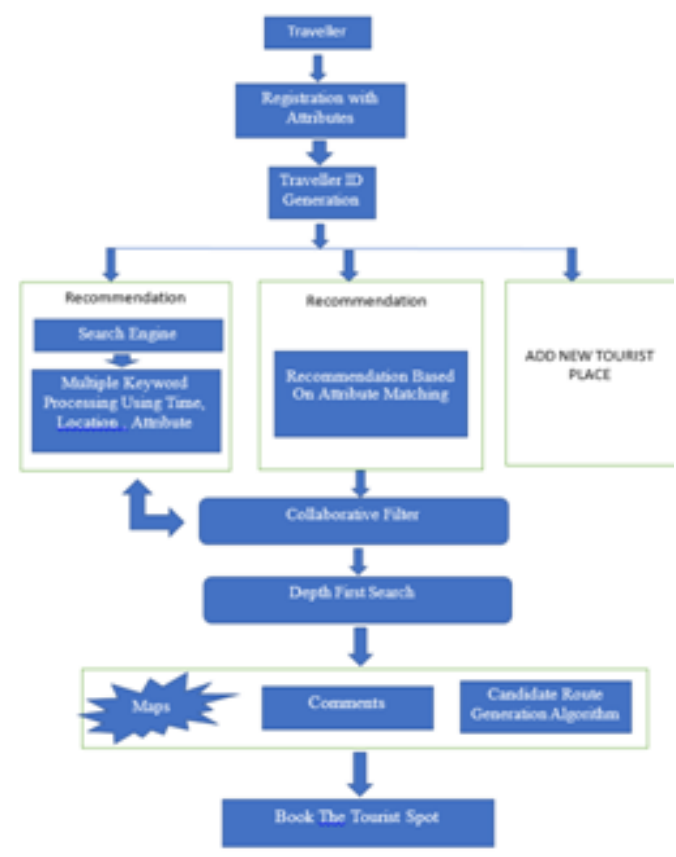

Fig:1 Diagram represents the architecture overview. 


\section{FUNCTIONALITIES}

\section{Keyword Extraction}

In this section, we tend to gift however we tend to extract the linguistics that means regarding the keywords including intending a resembled record to explain the level of association connecting keywords and results. The keyword removal module 1st counts abstraction, ephemeral credit rates for each keyword within the corpus. At question time, every question keyword are harmonised to pre-computed amount of matching.

\section{Geo-Specific Keywords}

Some labels distinct spot, that renders its abstraction type. To label the geo-specificity of any label, Associate in Nursing outer info identifies geo-terms within the overall patchset later tag allocation on the plan scales the known geo-terms. Precisely, to spot title labels, tend to leverage Associate in Nursing obvious geo-database. For a label, usingGDA, we tend to setGDA as one if latitude; longitude $\mathbf{P}$ is coming, and zero. Later, victimization the global dispersion of the labels,will realize place-level geo-terms similar 'Taipei 101' in clamant geo-terms. Nation type geo-terms and city type geo-terms are much more cosmopolitan on world than place type geo-terms. Consequently, we tend to work out the variance Geo Var of latitude; longitude P set together with tag w. With these options, we tend to outline (GS) score of a tag.

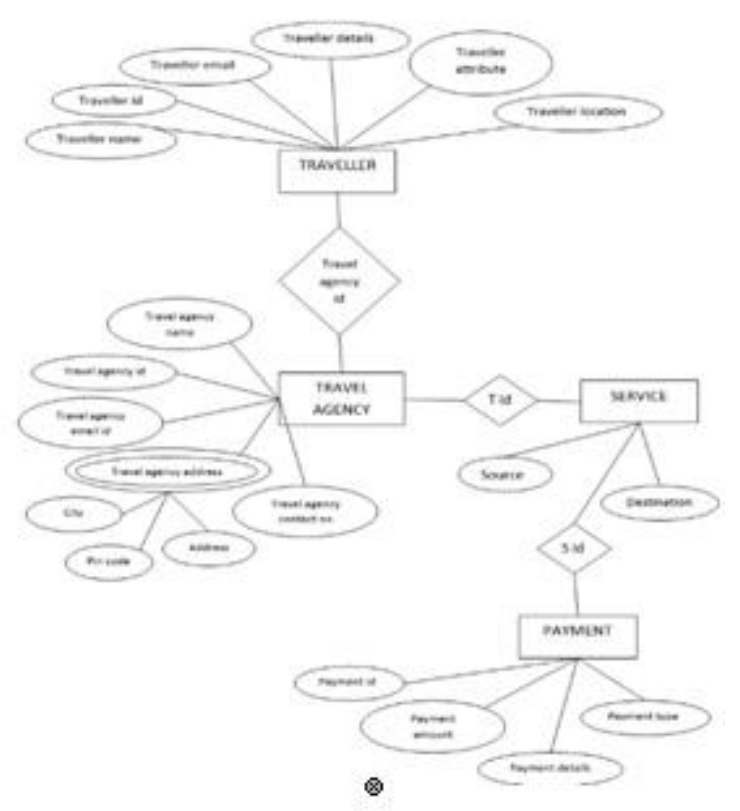

\section{Below Fig2: TRAVEL ER-DIAGRAM}

1.Personalized Tour Testimonial with POI Availability plus Dubious Traveling Time which was done by 'ChenyiZhang', 'HongweiLiang', 'KeWang',

'JianlingSun'.The fundamental they have created and appear to improve is the client's customized inclinations on POI's, the client's time spending that compels the complete voyaging and attending the time, the time window for the POI availability, the vulnerability of voyaging moment between POI's. using collaborative shifting makes it to the best part and the setting is to clarify just in the theoretical design as it were.

2. A circumstances conscious personalized tour implication practice based on geotagged communicative means data mining and developed by 'AbdulMajid', 'LingChen', 'GencaiChen', 'HamidTurabMirza', 'IbrarHussain' \& 'JohnWoodward'. In light of clients' voyaging inclinations inspired from their movement encounters uncovered via web-based networking media locales by sharing geotagged photographs, we propose another technique for prescribing traveller areas that comprise pertinent over clients while assigned setting. We get client explicit travel inclinations from his/her movement history in one city and utilize these to suggest visitor areas in another city. Used annotate locations with semantic. Using individual user data we recommend places. Photo based recommendation for travel is not efficient.

3. Voyage Direction through Mining People Attributes including Travel Group Types From Community-Contributed Photos by the

'YanYingChen', 'AnJungCheng', and 'WinstonHsu'. We propose to direct customized travel suggestion with extra granting explicit client profiles or attribute(e.g., gender, age, race)as well as movement bunch types (e.g., family, companions, couple). Rather than mining photograph logs just, we abuse the naturally distinguished individuals properties and travel bunch types in the photograph substance.

4. Travel Package Recommendation System is written by A Literature Review Himani M. Mishra, Dr Ms V. M. Deshmukh.This paper briefs about travel package recommender systems suggested by various sites and applications. Following is the audit of work done around there Multi-operator based recommender system. Collaborative Filtering \& amp, Content Aware Recommendation System, Based on Location Based Social Network yet the scenery is, It could have been additionally helping if there could be a touring plan proposal framework which could contain a succession of areas alongside plausible travel routes. There is much more to investigate and grow in this field, which can ease clients who like to investigate new places or like voyaging a great deal.

5. Hybrid Approach for Location-Based Customized POI Travel Recommendation System done by Chitra PS,Dr. Girijamma H A. The in turn of the examination give us the A movement proposal structure which centres around tweaked recommendations for clients. The labels just as geotags of pictures transferred by the clients on social locales like Flickr is influenced use so as to suggest the focal points (POI) to the clients. A hybrid approach with the use of tags, geotags and the owner (author) of the image is proposed in this paper. Client based community oriented separating is utilized to make proposals for the clients. The advantages of the proposed methodology are shown by trial examination on an 
extensive bunch of data, the disadvantage is to Existing suggestion frameworks experience the ill effects of an issue of sparsity. Sparsity issue manages lacking information identified with a client or any travel area.

6. "A Personalized Travel Recommender Model Based on Content-based Prediction and Collaborative Recommendation Shini Renjith, Anjali C", this research suggests that The community-oriented travel recommender portrays the design of a customized versatile travel indicator by performing content-based sifting and inductive learning procedures relying upon movement impressions. The savvy recommender is controlled by shared systems to determine the multiarmed logical outlaw issue of the investigation/misuse issue explorer the bad mark of this is Each visit of the client to any of these areas is recorded and fills in as a store that can be mined to recognize the individual's inclinations, which is an extraordinary test.

\section{TRAVEL TEST CASE}

The test case table shows each process execution of how the user data is stored and how it allows the user to pass the registered details and let the user in and admin manages the user's data from the backend storage. Admin validates the user and recommends places by the highest order of searching, all the results getting key, pass/fail can be seen on the web page of travel.

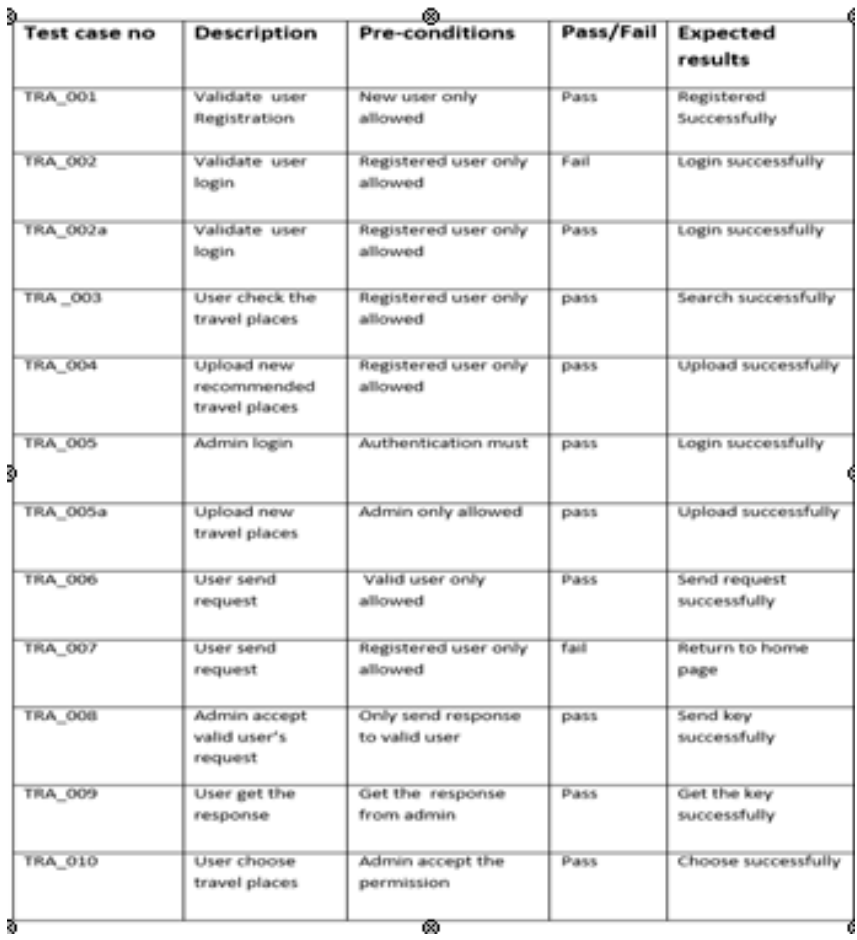

ICCF is assessed on an extensive range field-based informal organization dataset crept from Jiepang interpersonal organization. We select POIs that are attended by something like ten clients they have been to no less than ten unmistakable areas. At last, a sum of 265,951 clients and 189,850 points are then saved, and the thickness of these clients on these POIs.

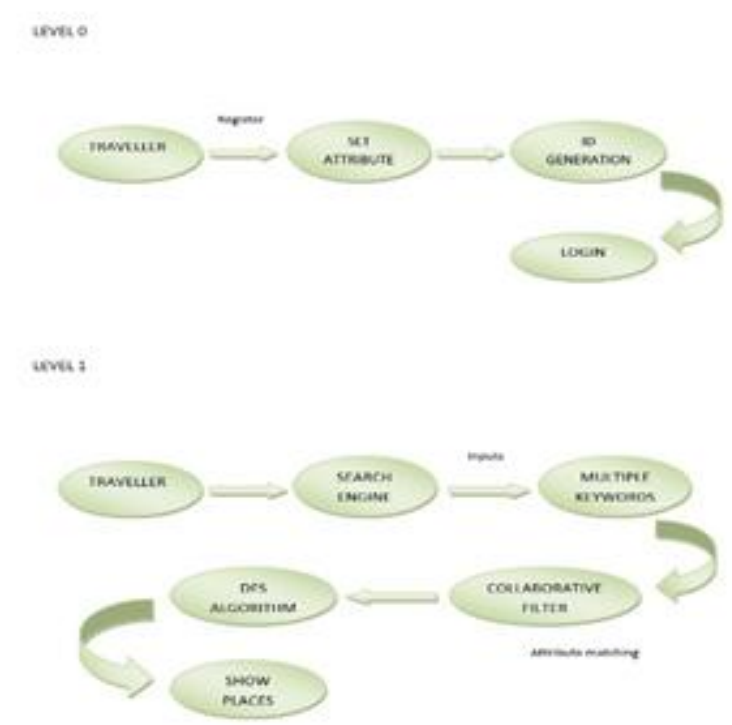

Fig 3:The Levels of recommendation for travel

\section{EXPERIMENT RESULTS}

Associations amidst baselines Each identification of ICCF were exhibited in above Fig. First, we have a tendency to perceive that ICCF exceeds LibFM by a major edge. one in all the explanations is that ICCF counts all un visited spots as contradiction however names lower conviction for contrary choice whereas LibFM solely inspects a number from them and treats them equally necessary as decisive ones. Besides, LibFM develops testimonial enforcement with an accumulating abundance of contradictory specimens. Depth-first search algorithm provides the highest order of searched places by the user.

\begin{tabular}{|c|c|}
\hline TRAVELER & ADMIN \\
\hline \multirow{2}{*}{$\begin{array}{l}\text { + Travelername } \\
\text { + TravelerID } \\
\text { + Travekeraddress } \\
\text { + Travelerdetals } \\
\text { + Interst plece } \\
\text { +Search places } \\
\text { + Vew nups } \\
\text { +Enter conmends } \\
\text { +Book places }\end{array}$} & $\begin{array}{l}\text { +AdrmintD } \\
\text { +Aderindetals } \\
\text { +Reconmendation places } \\
\text { + Add new places } \\
\text { +Vew traveler crits } \\
\text { +maintain database }\end{array}$ \\
\hline & \multirow{2}{*}{$\begin{array}{l}+ \text { +login() } \\
+ \text { Reconmend(0) } \\
+ \text { +Add) } \\
+ \text { +Vewd } \\
+ \text { logout() }\end{array}$} \\
\hline 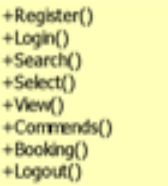 & \\
\hline
\end{tabular}

Fig: Travel class diagram

VII. DERIVED RESULTS

\section{TEXT MINING WITH MAP NAVIGATION:}

After getting the recommendation from another user, the user have to test the item and choose the item with the help of mining also the user has to choose the place by google map navigation by the most recommended place. Text digging customarily comprises specific manner like structuring the input text, determining models inside the structured, and certainly evaluation and understanding of the output. 


\section{CANDIDATEROUTE GENERATION ALGORITHM:}

The Candidate Route Generation formula is employed to mix completely diverse ways to extend the quantity and variety. These dissimilar applicant ways area unit created by linking the subsequences of trajectories. Here we conduce to inject the preprocessing methodology 1 st. we tend to then utilize the pre-processing results to accelerate the planned route reconstruction formula. The planned approach accounts for the distribution of transit demand on the $20+$ generated route and aims to reduce the distinction between route's overhead and revenue by prediction the dish.

\section{RECOMMENDATION AND RATING:}

Item-based cooperative filtering could be a model-based formula for creating recommendations. within the formula, the similarities between completely different things within the dataset area unit calculated by exploitation one in all variety of similarity measures, and so these same values area unit wont to predict ratings for user-item pairs not gift within the dataset. by exploitation this formula we've to predict the ratings of the item with the assistance another user's recommendation and suggestion concerning the item and place that is appropriate or not for the actual person. The higher than a formula that wont to calculate the very best rating concerning the item set and able to opt for that location to require a call by the US

\section{CONCLUSION:}

We intend an adequate travel recommendation framework to distinguish the multiple keywords and resemble the computation of paths and outlined a plot regeneration algorithm to aggregate route sections into journey routes in accordance with time, attribute and geo-specific location. Also, the traveller would be provided with distinctive paths to rise particular significance and heterogeneity using our system. FUTURE WORK:

Due to the real-time demands for online systems, we strive to diminish the reckoning expense by registering recurred queries furthermore to determine the comparative parameters automatically near eternity

\section{REFERENCES}

1. Z. Chen, H. T. Shen, X. Zhou, Y. Zheng, and X. Xie, "Searching trajectories by locations: An efficiency study," in Proc. ACM SIGMOD Int. Conf. Manage. Data, 2010, pp. 255-266.

2. H.-P. Hsieh and C.-T. Li, "Mining and planning time-aware routes from check-in data," in Proc. 23rd ACM Int. Conf. Conf. Inf. Knowl. Manage., 2014, pp. 481-490.

3. V. S. Tseng, E. H.-C. Lu, and C.-H. Huang, "Mining temporal mobile sequential patterns in location-based service environments," in Proc. Int. Conf. Parallel Distrib. Syst., 2007, pp. 1-8.

4. W. T. Hsu, Y. T. Wen, L. Y. Wei, and W. C. Peng, "Skyline travel routes: Exploring skyline for trip planning," in Proc. IEEE 15th Int. Conf. Mobile Data Manage., 2014, pp. 31-36.

5. Y. Zheng, L. Zhang, X. Xie, and W.-Y. Ma, "Mining interesting locations and travel sequences from GPS trajectories," in Proc. 18th Int. Conf. World Wide Web, 2009, pp. 791-800.
6. Q. Yuan, G. Cong, and A. Sun, "Graph-based point-of-interest recommendation with geographical and temporal influences," in Proc. 23rd ACM Int. Conf. Conf. Inf. Knowl. Manage., 2014, pp. 659-668.

7. M. Ye, P. Yin, W.-C. Lee, and D.-L. Lee, "Exploiting geographical influence for collaborative point-of-interest recommendation," in Proc. 34th Int. ACM SIGIR Conf. Res. Develop. Inf. Retrieval, 2011,pp 325-334.

8. Y.-T. Wen, P.-R. Lei, W.-C. Peng, and X.-F. Zhou, "Exploring socia influence on location-based social networks," in Proc. IEEE Int. Conf. Data Mining, 2014, pp. 1043-1048.

9. Y.-T. Wen, K.-J. Cho, W.-C. Peng, J. Yeo, and S.-W. Hwang, "KSTR Keyword-aware skyline travel route recommendation," in Proc. IEEE Int. Conf. Data Mining, 2015, pp. 449-458.

10. [10] Y. Tao, L. Ding, X. Lin, and J. Pei, "Distance-based representative skyline," in Proc. IEEE 25th Int. Conf. Data Eng., 2009, pp. 892-903.

11. Y.-T. Zheng, et al., "Tour the world: Building a web-scale landmark recognition engine," in Proc. IEEE Conf. Comput. Vis. Pattern Recog., 2009, pp. 1085-1092.

12. H. Gao, J. Tang, and H. Liu, "Exploring social-historical ties on location-based social networks," in Proc. 6th Int. AAAI Conf. Weblogs Social Media, 2012, pp. 114-121. 